\title{
Tuning a Model Drug Delivery System for Size and Loading Capacity
}

\author{
Alyssa Amato, Clifford E. Larrabee, Jr. \\ University of Cincinnati, Department of Science \& Health \\ Cincinnati, Ohio, USA \\ amatoal@mail.uc.edu; cliff.larrabee@uc.edu
}

\section{Extended Abstract}

Targeted drug delivery methods bring medication to diseased areas of a patient's body without affecting normal tissue. Nanoparticles can use ordinary cellular transport routes to deliver drugs to targeted sites because they fall into a size range similar to proteins and other biological macromolecules.[1] Blood vessels in tumors are underdeveloped and leaky, allowing passive transport of the drug carrier to the target cells. Tumors also lack efficient lymphatic drainage, allowing the buildup of the drug in the affected cells. This effect is called enhanced permeability and retention (EPR). [2] To work well, the nanocarriers must be large enough to avoid excretion through the kidneys ( $\gtrsim 5 \mathrm{~nm}$ diameter) but small enough to avoid clearing by the reticuloendothelial system( $\lesssim 100 \mathrm{~nm}$ diameter).[3] Here we show that the size and loading capacity of a micellar model system can be increased by combining an anionic cosurfactant with the primary ionic surfactant.

Three experiments were carried out for the comparison of micelle formation and solubilization of tetrabutylammonium 10-undecenoate (TBAU) without and with 1/2-equivalent of 10-undecen-1-ol (10-U). Apparent molar volumes were determined from density measurements. The addition of 10-U increased the apparent molar volume from $540 \mathrm{~cm}^{3} \mathrm{~mol}^{-1}$ to $615 \mathrm{~cm}^{3} \mathrm{~mol}^{-1}$. This is an increase of $14 \pm 6 \%$ and the difference is significant $(\mathrm{p}<0.01$ ). Hydrodynamic radii were estimated from viscosity measurements. The addition of 10-U increased the hydrodynamic radius from $3.18 \mathrm{~nm}$ to $3.93 \mathrm{~nm}$. This is an increase of $24 \pm 3 \%$ and the difference is significant $(\mathrm{p}<0.01)$. The loading capacity of the lipophilic dye, oil blue $\mathrm{n}$, was determined from visible light absorbance measurements at $597 \mathrm{~nm}$. The addition of 10-U increased the loading capacity by $33 \pm 16 \%$ and the difference was significant $(\mathrm{p}<0.01)$.

The apparent molar volume results indicate that the addition of the cosurfactant increases the volume of the micellar pseudo-phase. The viscosity results indicate the at least part of the increase in partial molar volume is due to the increased volume of individual micelles. The solubilization results indicate that the solubilization of the lipophilic dye is correlated with the increased volume of the micellar pseudo-phase and also with the average volume of the individual micelles. The differences in percentage increases among partial molar volume, hydrodynamic radius, and loading capacity are not statistically significant $(\mathrm{p}>0.05)$.

Our results point to a practical approach to tuning the size and loading capacity of a simple model micellar drug delivery system. Size dependent, passive targeting via the EPR effect is now possible with the model system. This gives us a valuable tool for the rational design of potential therapeutic agents.

\section{References}

[1] S. Bamrungsap, Z. Zhao, T. Chen, L. Wang, C. Li, T. Fu, et al., "Nanotechnology in therapeutics: a focus on nanoparticles as a drug delivery system," Nanomedicine, vol. 7, pp. 1253-1271, 2012.

[2] Y. Matsumura and H. Maeda, "A new concept for macromolecular therapeutics in cancer chemotherapy: mechanism of tumoritropic accumulation of proteins and the antitumor agent smancs," Cancer research, vol. 46, pp. 6387-6392, 1986.

[3] E. Blanco, H. Shen, and M. Ferrari, "Principles of nanoparticle design for overcoming biological barriers to drug delivery," Nature biotechnology, vol. 33, pp. 941-951, 2015. 